\title{
Atividade anticâncer de nanopartículas carregadas com bromelina: uma revisão
}

\section{sistemática}

\author{
Anti-cancer activity of bromelline-loaded nanoparticles: a systematic review \\ Actividad anticáncer de nanopartículas cargadas de bromelina: una revisión sistemática
}

Recebido: 17/11/2021 | Revisado: 26/11/2021 | Aceito: 04/12/2021 | Publicado: 13/12/2021

Eduardo Henrique Barros Ferreira

ORCID: https://orcid.org/0000-0002-0072-3378 Universidade Estadual do Ceará, Brasil E-mail: eh1405@hotmail.com

Francisco das Chagas Araújo Sousa

ORCID: https://orcid.org/0000-0001-7244-9729 Universidade Estadual do Piauí, Brasil E-mail: franciscoaraujo@ ccs.uespi.br

Hercília Maria Lins Rolim

ORCID: https://orcid.org/0000-0003-4469-6858 Universidade Federal do Piauí, Brasil

E-mail: hercilia.rolim@gmail.com

Karine Costa Melo

ORCID: https://orcid.org/0000-0001-8253-859X

Universidade Federal do Maranhão, Brasil

E-mail: karinemelo09@gmail.com

Wenderson Costa da Silva

ORCID: https://orcid.org/0000-0001-6031-9775

Universidade Estadual do Maranhão, Brasil

E-mail: wendersoncosta09@hotmail.com

Kelly Rose Pinho Moraes

ORCID: https://orcid.org/0000-0002-5784-4939

Universidade Estadual do Maranhão, Brasil

E-mail: kelly.rose125@gmail.com

Ana Tereza Santos Dias de Almeida

ORCID: https://orcid.org/0000-0001-9927-1836

Centro Universitário de Ciências e Tecnologia do Maranhão, Brasil

E-mail: aterezadias@gmail.com

Marconny Lira da Silva

ORCID: https://orcid.org/0000-0003-0334-9294

Centro Tecnológico de Ensino Múltiplo, Brasil

E-mail: marconnylirads@hotmail.com

Linccon Fricks Hernandes

ORCID: https://orcid.org/0000-0002-7642-3080 Escola Superior de Ciências da Santa Casa de Misericórdia de Vitória, Brasil E-mail: fricksjr@hotmail.com

Laila da Silva Mota Vilanova

ORCID: https://orcid.org/0000-0001-8873-5914 Centro Universitário de Ciências e Tecnologia do Maranhão, Brasil

E-mail: lailajoseneto@gmail.com

Thaiane Lima Rocha

ORCID: https://orcid.org/0000-0002-1007-0630

Centro Universitário Euro-Americano, Brasil

E-mail: thaiane.fisio@hotmail.com

Joelson da Silva Medeiros

ORCID: https://orcid.org/0000-0002-6339-2916

Centro Universitário Estácio, Brasil

E-mail: profjoelsonmedeiros@gmail.com

Mariana de Oliveira Sanchez

ORCID: https://orcid.org/0000-0002-4569-5497 Faculdade Estácio, Brasil

E-mail: profmarianasanchez@gmail.com

Apolo Kassio Barros da Silva

ORCID: https://orcid.org/0000-0003-0526-6169

Universidade Estadual do Maranhão, Brasil

E-mail: apolo19972009@gmail.com 


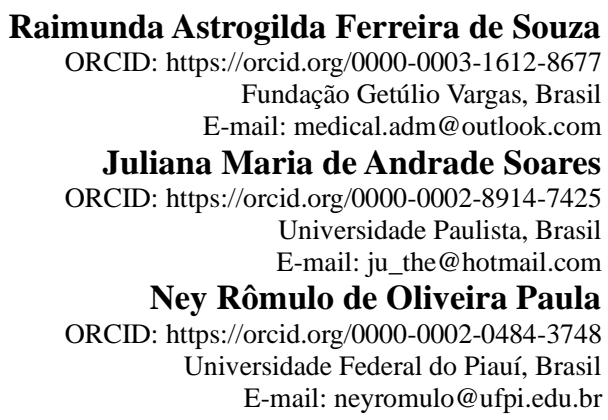

\title{
Resumo
}

O câncer é um grande problema de saúde pública e dentre os tratamentos disponíveis a quimioterapia é um dos mais utilizados, embora as drogas quimioterápicas sejam tóxicas para células cancerosas, elas apresentam especificidade baixa e, consequentemente, passam a ser tóxicas para as células saudáveis também. Uma estratégia que busca aumentar a eficácia terapêutica dos quimioterápicos e minimizar os efeitos adversos é a utilização de nanopartículas. Para tal, objetivou-se de modo geral revisar a literatura disponível a atividade anticâncer de nanopartículas carregadas com bromelina na área farmacêutica. Trata-se de uma revisão sistemática que teve como problemática "Qual a atividade anticâncer de nanopartículas carregadas com bromelina no campo da nanotecnologia farmacêutica?". Oito artigos foram incluídos nesta revisão, todos apresentaram abordagem quantitativa dos dados, em que $50 \%$ das publicações concentraram-se no ano de 2014 e 2016, todos disponíveis no idioma inglês; e a Índia foi o país com maior número de publicações $(50 \%)$. Este estudo permitiu observar que nanopartículas carregadas com bromelina apresenta potencial para atuar no tratamento de pacientes com câncer, tendo efeito antiproliferativo e antitumoral. Considera-se que novas pesquisas devem ser realizadas nesta linha de investigação, a fim de provar a necessidade encontrar novos tratamentos para o câncer e o potencial de atuação da bromelina.

Palavras-chave: Bromelaínas; Nanopartículas; Neoplasias; Terapêutica.

\begin{abstract}
Cancer is a major public health problem and among the treatments available, chemotherapy is one of the most used, although chemotherapy drugs are toxic for cancer cells, they have low specificity and, consequently, become toxic for healthy cells as well. A strategy that seeks to increase the therapeutic effectiveness of chemotherapy and minimize adverse effects is the use of nanoparticles. For this purpose, the objective was to generally review the available literature on the anticancer activity of nanoparticles loaded with bromelain in the pharmaceutical field. This is a systematic review that had the question "What is the anticancer activity of nanoparticles loaded with bromelain in the field of pharmaceutical nanotechnology?". Eight articles were included in this review, all of which presented a quantitative data approach, in which $50 \%$ of the publications were concentrated in the year 2014 and 2016 , all available in English; and India was the country with the highest number of publications (50\%). This study allowed us to observe that nanoparticles loaded with bromelain have the potential to act in the treatment of cancer patients, having an antiproliferative and antitumor effect. It is considered that further research should be carried out in this line of investigation, in order to prove the need to find new treatments for cancer and the action potential of bromelain.
\end{abstract}

Keywords: Bromelains; Nanoparticles; Neoplasms; Therapeutics.

\section{Resumen}

El cáncer es un importante problema de salud pública y entre los tratamientos disponibles, la quimioterapia es uno de los más utilizados, aunque los fármacos quimioterápicos son tóxicos para las células cancerosas, tienen baja especificidad y, en consecuencia, se vuelven tóxicos también para las células sanas. Una estrategia que busca incrementar la efectividad terapéutica de la quimioterapia y minimizar los efectos adversos es el uso de nanopartículas. Para ello, el objetivo fue revisar de manera general la literatura disponible sobre la actividad anticancerígena de nanopartículas cargadas con bromelina en el campo farmacéutico. Se trata de una revisión sistemática que tenía la pregunta "¿Cuál es la actividad anticancerosa de las nanopartículas cargadas con bromelina en el campo de la nanotecnología farmacéutica?”. En esta revisión se incluyeron ocho artículos, todos los cuales presentaron un enfoque de datos cuantitativos, en el que el 50\% de las publicaciones se concentraron en el año 2014 y 2016, todos disponibles en inglés; e India fue el país con el mayor número de publicaciones (50\%). Este estudio permitió observar que las nanopartículas cargadas con bromelina tienen el potencial de actuar en el tratamiento de pacientes con cáncer, teniendo un efecto antiproliferativo y antitumoral. Se considera que se deben realizar más investigaciones en esta línea de investigación, con el fin de demostrar la necesidad de encontrar nuevos tratamientos para el cáncer y el potencial de acción de la bromelina.

Palabras clave: Bromelaínas; Nanopartículas; Neoplasias; Terapéutica. 


\section{Introdução}

Segundo dados do Ministério da Saúde, o câncer foi responsável por mais de 230 mil óbitos no Brasil no ano de 2019 (Brasil, 2019). Esses dados mostram que essa patologia, atualmente, permanece como um grande problema de saúde pública, estando associada a transição epidemiológica, tecnológica, demográfica e a hábitos de vida.

Diante dos diversos tipos de câncer, os tratamentos básicos incluem cirurgia, radioterapia e o mais utilizado, a quimioterapia. A quimioterapia é realizada através de compostos químicos, chamados de quimioterápicos, no qual eles podem ser usados de forma isolada ou combinada com outros fármacos. Dependendo do tipo, duração, frequência e dosagem, esse tipo de tratamento acarreta muitos efeitos adversos ao organismo (Jesus et al., 2016; Wakiuchi, Marcon, Oliveira, \& Sales,2019).

Embora as drogas quimioterápicas sejam tóxicas para células cancerosas, elas apresentam especificidade baixa e, consequentemente, passam a ser tóxicas para as células saudáveis também. A explicação para isso é que os fármacos, em sua maioria, são pequenas moléculas que conseguem atravessar o endotélio em praticamente todas as partes do organismo, chegando aos seus alvos, mas também a outras regiões que não tem relação com a doença, provocando, assim, muitos efeitos adversos. Dentro dessa perspectiva, uma estratégia que busca aumentar a eficácia terapêutica dos quimioterápicos e minimizar os efeitos adversos é a utilização de nanopartículas, devido a seu tamanho, forma e carga superficial (Khawar et al., 2015; Mitragotri \& Lahann, 2012).

Existem diversas maneiras de desenvolver nanopartículas estruturadas para funcionarem como carregadores de medicamentos, entre eles estão as preparadas com materiais orgânicos, que possuem grandes vantagens por serem biocompatíveis e biodegradáveis. Um desses materiais orgânicos é a bromelina, que, segundo estudos, possui atividade anticancerígena e proteolítica (Marin et al., 2013; Pavan et al., 2012). A bromelina apresenta ainda grande potencial nutracêutico e importante ação mucolítica (Soares et al., 2021; Ferreira et al., 2021).

Sendo assim, este estudo teve como problemática "Qual a atividade anticâncer de nanopartículas carregadas com bromelina?". Para tal, objetivou-se de modo geral revisar a literatura disponível a atividade anticâncer de nanopartículas carregadas com bromelina, e de modo específico descrever a ação proteolítica da bromelina e caracterizar as nanopartículas.

Desta forma, a escolha da temática teve início a partir da necessidade de se compreender a atividade anticâncer de nanopartículas carregadas com bromelina nanoestruturada. Neste sentido, a relevância do trabalho foi apresentar as potencialidades da bromelina para o tratamento de câncer e evidenciar a necessidade de mais pesquisas na área em questão.

\section{Metodologia}

Trata-se de uma revisão sistemática da literatura conduzida conforme as recomendações PRISMA (Moher Moher et al.,). Este procedimento foi escolhido por possibilitar a síntese e análise do conhecimento científico já produzido sobre o tema "Atividade anticâncer de nanopartículas carregadas com bromelina: uma revisão sistemática".

O tema, determinou a construção da estratégia PICO, que representa um acrônimo para Problema (P), Intervenção (I), Comparação (C) e Desfechos (O-outcomes), na qual foi utilizada para a geração da questão norteadora desta revisão sistemática da literatura: "Quais evidências científicas mostram a atividade anticâncer de nanopartículas carregadas com bromelina?".

Para a localização dos estudos relevantes, que respondessem à pergunta de pesquisa, utilizou-se de descritores indexados e não indexados (palavras-chave) no idioma inglês. Os descritores foram obtidos a partir do Medical Subject Headings (MESH), dos Descritores em Ciências da Saúde (DeCS), como mostra o Quadro 1. 
Quadro 1. Elementos da estratégia PICO, descritores e palavras-chave utilizados. Teresina, PI, Brasil, 2021.

\begin{tabular}{|c|c|c|c|c|}
\hline \multirow[t]{2}{*}{$\begin{array}{l}\text { Objetivo/ } \\
\text { Problema }\end{array}$} & \multicolumn{4}{|c|}{ Quais evidências científicas mostram a atividade anticâncer de nanopartículas carregadas com bromelina? } \\
\hline & $\mathbf{P}$ & I & $\mathbf{C}$ & $\mathbf{O}$ \\
\hline $\begin{array}{c}\text { Extração } \\
\text { (Elementos) }\end{array}$ & Câncer & $\begin{array}{l}\text { Nanopartículas } \\
\text { carregadas com } \\
\text { bromelina }\end{array}$ & - & Agente Anticâncer \\
\hline $\begin{array}{l}\text { Conversão } \\
\text { (Descritor } \\
\text { controlado) }\end{array}$ & Neoplasms & Bromelains & - & $\begin{array}{l}\text { Antineoplastic Agents } \\
\text { OR Nanotechnology }\end{array}$ \\
\hline $\begin{array}{l}\text { Combinação } \\
\text { (Descritor não } \\
\text { controlado) }\end{array}$ & Cancer OR Tumor & Bromelain & - & "Technology, Pharmaceutical” \\
\hline Construção & $\begin{array}{l}\text { (Neoplasms OR Cancer } \\
\text { OR Tumor) }\end{array}$ & $\begin{array}{l}\text { Bromelains OR } \\
\text { Bromelain }\end{array}$ & - & $\begin{array}{c}\text { "Antineoplastic Agents" OR Nanotechnology } \\
\text { OR "Technology, Pharmaceutical" }\end{array}$ \\
\hline Uso & $\begin{array}{l}\text { (Neoplasms OR Cancer } \\
\text { nanotechnology OR "Tec }\end{array}$ & $\begin{array}{l}\text { Tumor) AND } \\
\text { gy, Pharmaceutic }\end{array}$ & & romelain) AND ("Antineoplastic Agents" OR \\
\hline
\end{tabular}

Fonte: Descritores Decs e Mesh (2021).

O elemento $\mathrm{C}$ da estratégia PICO não foi abordado nesta pesquisa pois esta não tem por objetivo comparar intervenções. Por meio de descritores e palavras-chave foi realizada uma busca eletrônica avançada nas bases de dados da National Library of Medicine; e na Web of Science, conforme demonstrado no quadro 2.

Quadro 2. Estratégias de busca utilizadas nas bases de dados PUBMED e Web of Science. Teresina, PI, Brasil, 2021.

\begin{tabular}{|c|l|c|c|c|}
\hline Base de dados & \multicolumn{1}{|c|}{ Estratégia de Busca } & $\begin{array}{c}\text { Resultado } \\
\text { s }\end{array}$ & $\begin{array}{c}\text { Filtrados } \\
\text { PUBMED }\end{array}$ & $\begin{array}{c}\text { Selecionado } \\
\text { s }\end{array}$ \\
& $\begin{array}{l}\text { ("Neoplasms OR Cancer OR Tumor) AND (Bromelains)) AND } \\
\text { ("Antineoplastic Agents" OR Nanotechnology OR "Technology, } \\
\text { Pharmaceutical") }\end{array}$ & 56 & 24 & 4 \\
\hline Web of Science & $\begin{array}{l}\text { (ALL=((Neoplasms OR Cancer OR Tumor))) AND ALL=(Bromelains } \\
\text { OR Bromelain) }\end{array}$ & 184 & 83 & 4 \\
\hline
\end{tabular}

Fonte: Web of Science e PUBMED (2021).

Como critérios de inclusão utilizaram-se estudos disponíveis em sua totalidade, publicados nos últimos oito anos, de 2014 até 2021, nos idiomas Português, Espanhol e Inglês. Foram excluídos da busca inicial capítulos de livros, resumos, textos incompletos, teses, dissertações, monografias, relatos técnicos e outras formas de publicação que não fossem artigos científicos completos.

A análise para seleção dos estudos foi realizada em duas fases, a saber:

$\mathrm{Na}$ primeira, os estudos foram pré-selecionados segundo os critérios de inclusão e exclusão e de acordo com a estratégia de funcionamento e busca de cada base de dados.

Na segunda fase os estudos foram analisados quanto ao potencial de participação no estudo, avaliando o atendimento à questão de pesquisa, bem como o tipo de investigação, objetivos, amostra, método, desfechos e conclusão, resultando em oito (08) artigos.

Ao final oito (08) artigos atenderam a questão norteadora e foram adicionados ao estudo, conforme demonstrando na Figura 1 . 
Figura 1. Fluxograma de seleção de artigos. Teresina, PI, Brasil, 2021.

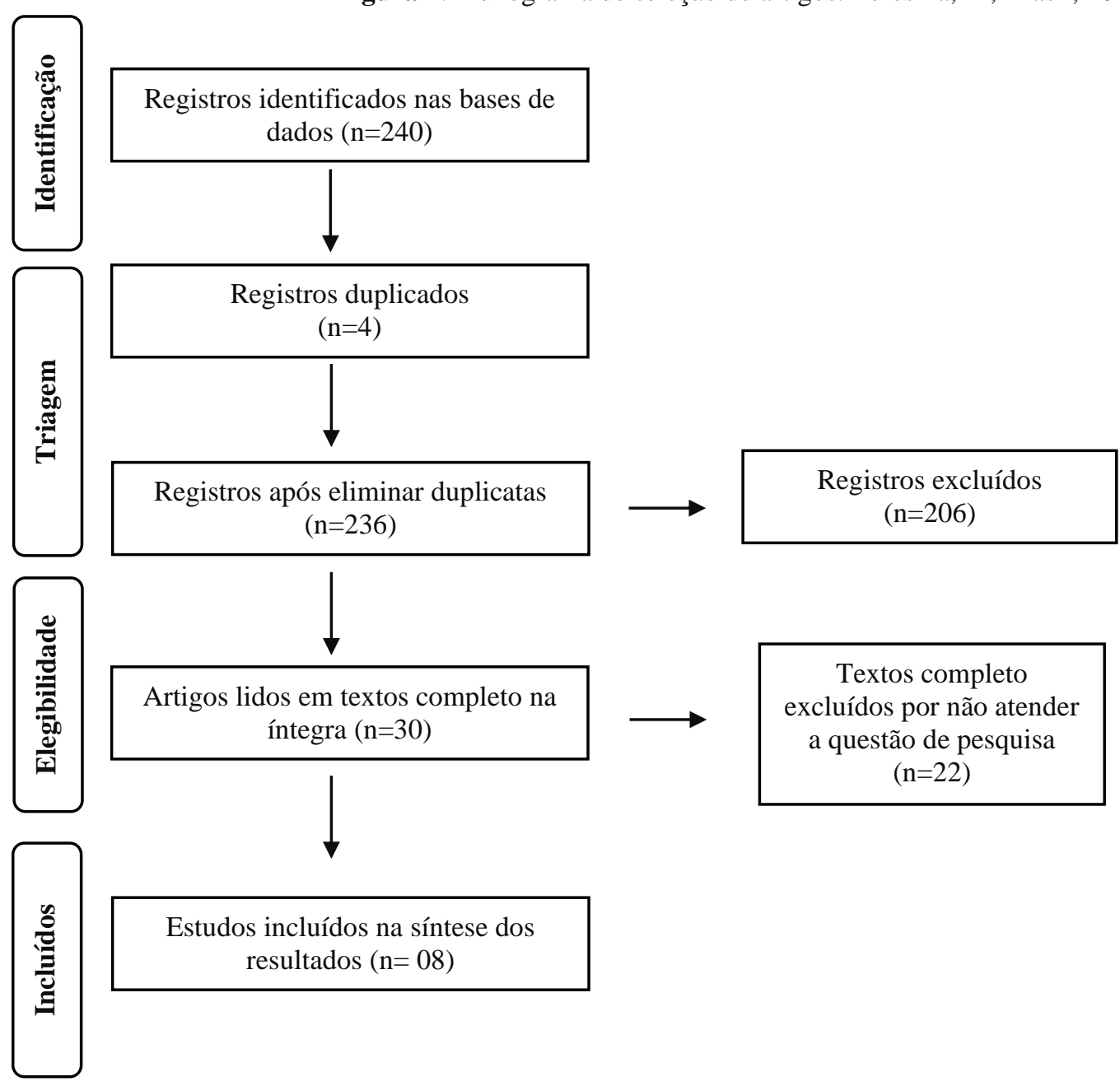

Fonte: Bases de dados Web of Science e PUBMED (2021).

Quanto a análise dos dados, foram criadas categorias analíticas que facilitou a ordenação e a sumarização de cada estudo. Essa categorização foi realizada de forma descritiva, indicando os dados mais relevantes para o estudo.

A pesquisa levou em consideração os aspectos éticos dos estudos quanto às citações, respeitando a autoria das ideias, os conceitos e as definições presentes nos artigos incluídos na revisão.

Optou-se pela análise em forma estatística e de forma de texto, utilizando cálculos matemáticos e inferências, que serão apresentados em quadros e tabelas para facilitar a visualização e compreensão.

\section{Resultados e Discussão}

Na base PUBMED, como busca total foram encontrados cinquenta e seis (56) estudos, aplicando na pesquisa o filtro que limita por texto completo dos últimos cinco anos, obteve-se vinte e quatro (24) estudos, estes foram analisados títulos e resumos, onde obteve-se catorze (14) artigos para análise completa na íntegra, e teve como resultado final de quatro (4) estudos condizentes com a questão de pesquisa após a análise.

$\mathrm{Na}$ Web of Science foram obtidos cento e oitenta e quatro estudos (184) estudos como busca geral, sendo que limitando a busca para artigos com texto completo realizado nos últimos cinco anos, obteve-se oitenta e três (83) estudos, estes foram analisados títulos e resumos, resultando em dezesseis (16) estudos para análise completa na íntegra, onde quatro (4) estudos foram condizentes com a questão desta pesquisa e foram adicionados ao estudo. 
Dos oito (08) artigos incluídos nesta revisão, todos apresentaram abordagem quantitativa dos dados, em que $50 \%$ das publicações concentraram-se no ano de 2014 e 2016, os demais anos apresentaram 12,5\% cada, respectivamente. Quanto ao idioma todos os artigos estavam na língua inglesa; em que a Índia foi o país com maior número de publicações (50\%). Todos os periódicos apresentaram o mesmo percentual de ocorrência, com cerca de 12,5\% cada, conforme demonstrado na Tabela 1.

Tabela 1. Análise descritiva das produções científicas acerca da atividade anticâncer de nanopartículas carregadas com bromelina. Teresina, PI, Brasil, 2021. $(\mathrm{N}=08)$

\begin{tabular}{lll}
\hline Variáveis & $\mathbf{N}$ & \% \\
\hline Abordagem do estudo & 08 & 50,0 \\
Quantitativo & & 25,0 \\
Ano & 02 & 25,0 \\
$\mathbf{2 0 1 4}$ & 02 & 12,5 \\
$\mathbf{2 0 1 6}$ & 01 & 12,5 \\
$\mathbf{2 0 1 7}$ & 01 & 12,5 \\
$\mathbf{2 0 1 8}$ & 01 & 12,5 \\
$\mathbf{2 0 1 9}$ & 01 & 100,0 \\
$\mathbf{2 0 2 0}$ & & 12,5 \\
Idiomas & 08 & 50,0 \\
Inglês & & 12,5 \\
País & & 25,0 \\
Itália & 01 & 12,5 \\
Índia & & 12,5 \\
Brasil & 04 & 12,5 \\
Phina & 01 & 12,5 \\
ACS Nańdicos & 02 & 12,5 \\
Journal of Biomedical Nanotechnology & & 12,5 \\
Pharmaceutical Research & 01 & 12,5 \\
Materials Science \& Engineering C & 01 & 12,5 \\
International Journal of Biological Macromolecules & 01 & 01 \\
Scientific Reports & & \\
Colloids and Surfaces B: Biointerfaces & 01 & 01 \\
\hline
\end{tabular}

Fonte: Dados da pesquisa (2021).

O Quadro 3 mostra a distribuição dos estudos segundo autores e anos de publicação, título e objetivo principal. Diferentes estudos com abordagens experimentais versaram sobre a atividade anticâncer de nanopartículas carregadas com bromelina. 
Quadro 3. Publicações incluídas segundo o título do artigo, autor e o objetivo principal. Teresina, PI, Brasil. 2021. (N=08).

\begin{tabular}{|c|c|c|c|}
\hline $\begin{array}{c}\mathbf{N}^{\circ} \text { de } \\
\text { ordem e } \\
\text { Base }\end{array}$ & Título do artigo & Autor/ano & Delineamento de Pesquisa \\
\hline $\begin{array}{c}\text { A1 } \\
\text { PUBMED }\end{array}$ & $\begin{array}{l}\text { Bromelain Surface Modification } \\
\text { Increases the Diffusion of Silica } \\
\text { Nanoparticles in the Tumor Extracellular } \\
\text { Matrix }\end{array}$ & $\begin{array}{l}\text { Parodi et al. } \\
\text { (2014) }\end{array}$ & $\begin{array}{l}\text { Mostrar que a atividade proteolítica da Bromelina pode } \\
\text { ser transferida para a superfície de nanopartículas de } \\
\text { sílica mesoporosa (MSN) aumentando efetivamente sua } \\
\text { difusão dentro da matriz extracelular tumoral (ECM). }\end{array}$ \\
\hline $\begin{array}{c}\text { A2 } \\
\text { PUBMED }\end{array}$ & $\begin{array}{llr}\begin{array}{l}\text { Anti-Cancer Activity } \\
\text { melainNanoparticles }\end{array} & \text { by } & \text { Bro } \\
\text { Administration } & & \text { Oral } \\
\end{array}$ & $\begin{array}{c}\text { Bhatnagar et al. } \\
\text { (2014) }\end{array}$ & $\begin{array}{l}\text { Analisar a atividade anticâncer de nanopartículas de } \\
\text { bromelina por administração oral. }\end{array}$ \\
\hline $\begin{array}{c}\text { A3 } \\
\text { PUBMED }\end{array}$ & $\begin{array}{l}\text { Bromelain-Functionalized Multiple-Wall } \\
\text { Lipid-Core Nanocapsules: Formulation, } \\
\text { Chemical Structure and Antiproliferative } \\
\text { Effect Against Human Breast Cancer } \\
\text { Cells (MCF-7) }\end{array}$ & $\begin{array}{l}\text { Oliveira et al. } \\
\text { (2016) }\end{array}$ & $\begin{array}{l}\text { Conduzir uma abordagem para funcionalização de } \\
\text { superfície desenvolvida para nanocápsulas de núcleo } \\
\text { lipídico para tratar tumores sólidos. }\end{array}$ \\
\hline $\begin{array}{c}\text { A4 } \\
\text { PUBMED }\end{array}$ & $\begin{array}{l}\text { Hyaluronic acid grafted PLGA copolymer } \\
\text { nanoparticles enhance the targeted } \\
\text { delivery of Bromelain in Ehrlich's } \\
\text { Ascites Carcinoma }\end{array}$ & $\begin{array}{l}\text { Bhatnagar, Pant, } \\
\text { Shukla, Panda } \\
\text { \& Gupta (2016) }\end{array}$ & $\begin{array}{l}\text { Analisar a eficácia antitumoral de Bromelina (BL) e a } \\
\text { capacidade de direcionamento dessas nanopartículas } \\
\text { (NPs) de copolímero BL em células cancerosas que } \\
\text { expressam diferentes níveis de receptores de CD44. }\end{array}$ \\
\hline $\begin{array}{c}\text { A5 } \\
\text { Web of } \\
\text { Science }\end{array}$ & $\begin{array}{l}\mathrm{pH} \text {-sensitive bromelain nanoparticles by } \\
\text { ortho ester crosslinkage for enhanced } \\
\text { doxorubicin penetration in solid tumor }\end{array}$ & $\begin{array}{l}\text { Wang, } \mathrm{Xu}, \mathrm{Xu}, \\
\text { Fang, \& Tang } \\
(2020) \\
\end{array}$ & $\begin{array}{l}\text { Analisar nanopartículas de bromelina sensíveis ao } \mathrm{pH} \text { por } \\
\text { reticulação orto éster para aumentar a penetração da } \\
\text { doxorrubicina em tumor sólido. }\end{array}$ \\
\hline $\begin{array}{c}\text { A6 } \\
\text { Web of } \\
\text { Science }\end{array}$ & $\begin{array}{l}\text { Bromelain-immobilized and lactobionic } \\
\text { acid-modified chitosan nanoparticles for } \\
\text { enhanced drug penetration in tumor } \\
\text { tissues }\end{array}$ & $\begin{array}{l}\text { Wang et al. } \\
\text { (2018) }\end{array}$ & $\begin{array}{l}\text { Investigar a atividade proteolítica de nanopartículas } \\
\text { imobilizados com bromelina em esferoides multicelulares } \\
\text { semelhantes a tumor. }\end{array}$ \\
\hline $\begin{array}{c}\text { A7 } \\
\text { Web of } \\
\text { Science }\end{array}$ & $\begin{array}{l}\text { Cisplatin bioconjugated enzymatic GNPs } \\
\text { amplify the efect of cisplatin with } \\
\text { acquiescence }\end{array}$ & $\begin{array}{l}\text { Iram et al. } \\
\text { (2019) }\end{array}$ & $\begin{array}{l}\text { Sintetizar nanopartículas de ouro monodispersas usando } \\
\text { protease de cisteína à base de abacaxi, bromelina, como } \\
\text { redutor e também como agente de funcionalização de } \\
\text { superfície. }\end{array}$ \\
\hline $\begin{array}{c}\text { A8 } \\
\text { Web of } \\
\text { Science }\end{array}$ & $\begin{array}{l}\text { Gold nanoconjugates } \begin{array}{r}\text { reinforce } \\
\text { potency of conjugated } \\
\text { doxorubicin }\end{array} \\
\text { dotatin and }\end{array}$ & $\begin{array}{l}\text { Iram et al. } \\
\quad(2017)\end{array}$ & $\begin{array}{l}\text { Investigar o aumento e o efeito sinérgico das } \\
\text { nanopartículas de ouro encapsuladas com bromelina } \\
\text { (protease de cisteína) combinadas exclusivamente CIS e } \\
\text { DOX (CIS e DOX conjugadas com B-AuNPs). }\end{array}$ \\
\hline
\end{tabular}

Fonte: Artigos pesquisados (2021).

O câncer é um problema de saúde pública com altos indicies mundialmente, portanto é de extrema importância o desenvolvimento de estudos na área, inclusive de novas abordagens tecnológicas que são essenciais para descoberta de novas terapias farmacológicas e tratamentos, e uma dessas abordagens é a utilização de nanopartículas com bromelina (Oliveira et al. 2016).

Existem muitas formas e utilização das nanopartículas, sendo uma delas o uso das nanopartículas de ouro com bromelina que são alvos de estudos experimentais em células tumorais, pois apresentam propriedades de grande proporção de volume permitindo um amplo número de moléculas de drogas anticancerígenas sendo entregues diretamente as células cancerosas (Iram et al. 2017), enquanto que a bromelina estabelece um papel de agente redutor e de cobertura, sintetizando as nanopartículas de ouro nos tamanhos desejados e é uma ótima escolha na redução do cloreto áurico e contribui na formação de uma corona estável na superfície das nanopartículas de ouro (Iram et al. 2019)

Além disso, possuem capacidade de direcionamento ativo, ou seja, a entrega dos fármacos é específica ao local do tumor, aumentando o reconhecimento de células cancerosas e absorção dos fármacos. As nanopartículas também podem se reunir passivamente na área do tumor devido ao aumento do efeito de permeação e retenção, consequentemente, aumenta a concentração intratumoral da droga e melhora a eficácia quimioterápica reduzindo os efeitos colaterais causados por drogas anticâncer (Wang et al. 2018).

Outras abordagens incluem nanocápsulas de núcleo lipídico de parede múltipla funcionalizadas com bromelina (Oliveira et al. 2016), nanopartículas com base em quitosana modificada com ácido lactobiônico (CL NPs) e posteriormente 
revestido com bromelina (Wang et al. 2018), nanopartículas de sílica mesoporosa com superfície proteolítica conjugada com bromelina (Parodi et al. 2014), nanopartículas de copolímero ácido láctico e ácido glicólico enxertadas com ácido hialurônico (Bhatnagar et al. 2016; Bhatnagar et al. 2014), e as nanopartículas sensíveis ao pH preparadas por reticulação de bromelina e com um agente de reticulação à base de orto éster (Wang et al. 2020),

Para a resultados eficientes é necessário considerar as características físicas e interfaciais das nanopartículas quanto a carga superficial, forma, tamanho e hidrofobicidade, é importante destacar que as nanopartículas são reconhecidas no organismo por opsoninas, como as proteínas do soro sanguíneo, componente do complemento (C3, C4 e C5) e imunoglobulina (IgG e IgM) (Iram et al. 2017).

Por terem uma grande diversidade no que se refere a biodistribuição, farmacocinética e mecanismo de transporte, há uma grande dificuldade em definir a dosagem das drogas anticâncer, e consequentemente, há um pobre acúmulo delas nas células cancerosas (Iram et al., 2017).

Atualmente, a matriz extracelular do tumor (MEC) é uma das principais barreiras que dificultam a disseminação das drogas em seu parênquima, diante disso, muitos pesquisadores passaram a estudar a bromelina como um meio capaz de penetrar essa barreira. Entretanto, a fim de obter a eficácia terapêutica desejada, é necessário que ela seja agrupada a nanopartículas (Bhatnagar et al., 2014; Bhatnagar et al., 2016).

Segundo Wang et al. (2020), deteriorar a matriz extracelular do tumor utilizando bromelina como forma de garantir uma maior penetração da droga e maiores efeitos antitumorais é uma técnica interessante, contudo por ter uma fraca estabilidade e uma potencial imunogenicidade, a bromelina precisa de um nanocarreador para que para que assim ela mantenha sua atividade proteolítica e a estratégia seja eficiente.

Em seu estudo, Parodi et al. (2014) utilizaram nanopartículas de sílica mesoporosas com bromelina para adentrar o parênquima tumoral in vivo e in vitro de câncer de mama e demonstraram que essa junção foi capaz de aumentar a absorção de partículas no endotélio, macrófagos e linhas de células cancerosas com impacto mínimo na viabilidade celular. Semelhante a isso, Wang et al. (2018) ao comparar nanopartículas de ácido lactobiônico (LA) puras e nanopartículas de ácido lactobiônico (LA) juntamente com bromelina como carreadores de doxorrubicina, provaram que com a bromelina havia uma maior capacidade de penetração no tumor e maior difusão das nanopartículas na área, o que levava a uma maior concentração do fármaco.

Além de sua característica proteolítica, a bromelina também tem poder redutor e serve como agente de cobertura, ou seja, ela consegue diminuir os efeitos colaterais e a toxicidade das nanopartículas, fazendo com que os medicamentos cheguem especificamente no núcleo das células cancerosas (Iram et al., 2017).

Neste aspecto, a ação anticâncer de nanopartículas com bromelina tem sido tem se mostrado promissora para atuar em células cancerígenas (Oliveira et al., 2017). Corroborando com os achados, Iram et al. (2019) afirmam que a bromelina é digerida pelo organismo sem perda da atividade biológica, se tornando importante para possíveis tratamentos de câncer devido a sua protease.

Já o estudo in vivo realizado por Bhatnagar et al. (2014) evidenciou que, a bromelina após o encapsulamento foi efetiva para utilização por via oral. Neste ponto, foi observado que os camundongos tiveram uma diminuição da carga tumoral do carcinoma e também um aumento da expectativa de vida. No qual, Parodi et al. (2014) afirmam que a bromelaína apresenta potencial para difusão de tumores, onde há evidencias ainda que, a presença da bromelina facilita a penetração de nanopartículas nas células tumores e aumenta a concentração dos medicamentos, tendo efeito antitumoral (Wang et al., 2018).

É visto que o efeito combinado da cisplatina (CIS) e doxorubicina (DOX) bioconjugadas de bromelaína encapsuladas em nanopartículas de ouro encapsuladas (B-AuNPs conjugadas CIS e DOX) também mostrou potencial no tratamento de osteossarcoma que foi confirmada utilizando através de espectroscopia UV-vis (Iram et al., 2017). E Bhatnagar et al. (2016) 
discorrem que a bromelina encapsulada em copolímero (nanopartículas bl-copolímeras) demonstrou supressão no crescimento tumoral de camundongos, onde Wang et al. (2020) expressam que o encapsulamento é efetivo já que a própria bromelina apresenta ação antiproliferativa.

\section{Considerações Finais}

Este estudo permitiu observar a atividade anticâncer de nanopartículas carregadas com bromelina, onde foi expresso que a bromelina apresenta potencial para atual no tratamento de pacientes com câncer, tendo efeito antiproliferativo e antitumoral.

A bromelina é uma substância muito estudada devido as suas propriedades nutracêuticas que incluem ação antioxidante e anti-inflamatória, e sua ampla utilização na área da saúde e indústria alimentícia, onde é notado que sua utilização vai desde o foco central do tratamento até a atuação como adjuvante para diversos medicamentos, sendo usada ainda, para o desbridamento enzimático. Portanto, deve-se explorar toda a sua potencialidade.

Como limitações do estudo tem-se o fato de não haver pesquisas suficientes para apoiar que os testes sejam realizados em humanos sem oferecer riscos à saúde. Por fim, a pesquisa possibilitou expandir os conhecimentos acerca da utilização da bromelina, e atividade anticâncer de nanopartículas de bromelina. Dessa forma, considera-se que novas pesquisas devem ser realizadas nesta linha de investigação, a fim de provar a necessidade encontrar novos tratamentos para o câncer e o potencial de atuação da bromelina.

\section{Referências}

Bhatnagar, P., Patnaik, S., Srivastava, A. K., Mudiam, M. K. R., Shukla, Y., Panda, A. K., \& Gupta, K. C. (2014). Anti-Cancer Activity of Bromelain Nanoparticles by Oral Administration. J. Biomed. Nanotechnol., 10, 3558-3575.

Bhatnagar, P., Pant, A. B., Shukla, Y., Panda, A., \& Gupta, K. C. (2016). Hyaluronic acid grafted PLGA copolymer nanoparticles enhance the targeted delivery of Bromelain in Ehrlich's Ascites Carcinoma. European Journal of Pharmaceutics and Biopharmaceutics, 105, $176-192$.

Brasil. (2019). Estatísticas de Câncer. Ministério da Saúde.

Ferreira, E. H. B., Melo, K. C., Da Silva, C. O., Da Silva, J. S., Vilanova, L. D. S. M., Siqueira, F. F. F. S., \& Paula, N. R. D. O. (2021). Ação Mucolítica da Bromelina: uma revisão integrativa. Research, Society and Development, 10(13).

Iram, S., Zahera, M., Wahid, I., Baker, A., Raish, M., Khan, A., \& Khan, M. S. (2019). Cisplatin bioconjugated enzymatic GNPs amplify the efect of cisplatin with acquiescence. Scientific Reports, 9.

Iram, S., Zahera, M., Khan, S., Khan, I., Syed, A., Ansary, A. A., . . \& Khan, M. S. (2017). Gold nanoconjugates reinforce the potency of conjugated cisplatin and doxorubicin. Colloids and Surfaces B: Biointerfaces, 160, 254-264.

Jesus, L. G., Cicchelli, M., Martins, G. B., Pereira, M. C. C., Lima, H. S., \& Medrado, A. R. A. P. (2016). Repercussões orais de drogas antineoplásicas: uma revisão de literatura. $R F O$, Passo Fundo, 21(1), 130-135.

Khawar, I. A., Kim, J. H., \& Kuh, H. J. (2015). Improving drug delivery to solid tumors: priming the tumor microenvironment. Journal of controlled release, 201, 78-89.

Marin, E., Briceno, M. I., \& Caballero-George, C. (2013). Critical evaluation of biodegradable polymers used in nanodrugs. Int J Nanomed, 8, $3071-3091$.

Mitragotri, S., \& Lahann, J. (2012). Materials for Drug Delivery: Innovative Solutions to Address Complex Biological Hurdles. Adv. Mater., 24, 3717-3723.

Moher, D., Liberati, A., Tetzlaff, J., \& Altman, D. G. (2009). Preferred reporting items for systematic reviews and meta-analyses: the prisma statement. BMJ, 339(211), 2535-2535.

Oliveira, C. P., Prado, W. A., Lavayen, V., Büttenbender, S. L., Beckenkamp, A., Martins, B. S., \& Pohlmann, A. R. (2017). Bromelain-Functionalized Multiple-Wall Lipid-Core Nanocapsules: Formulation, Chemical Structure and Antiproliferative Effect Against Human Breast Cancer Cells (MCF-7). Pharmaceutical Research, 34, 438-452.

Parodi, A., Haddix, S. G., Taghipour, N., Scaria, S., Taraballi, F, Cevenini, A., \& Tasciotti, E. (2014). Bromelain Surface Modification Increases the Diffusion of Silica Nanoparticles in the Tumor Extracellular Matrix. ACS nano, 8(10), 9874-9883.

Pavan, R., Jain, S., Shraddha, \& Kumar, A. (2012). Properties and Therapeutic Application of Bromelain: A Review. Biotechnol. Res. Int., $2012,1-6$. 
Research, Society and Development, v. 10, n. 16, e325101623454, 2021

(CC BY 4.0) | ISSN 2525-3409 | DOI: http://dx.doi.org/10.33448/rsd-v10i16.23454

Soares, A. N., Melo, K. C., Da Silva, W. C., Da Silva, C. O., Da Silva, R. A., Da Silva, K. G. S., \& Souza, F. D. C. A. (2021). Potenciais efeitos imunomoduladores de nutracêuticos no tratamento de Covid-19. International Journal of Development Research, 11(4), 46233-46238.

Wakiuchi, J., Marcon, S. S., Oliveira, D. C., \& Sales, C. A. (2019). A quimioterapia sob a ótica da pessoa com câncer: uma análise estrutural. Texto \& Contexto Enfermagem, 28.

Wang, X., Xu, J., Xu, X., Fang, Q., \& Tang, R. (2020). pH-sensitive bromelain nanoparticles by ortho ester crosslinkage for enhanced doxorubicin penetration in solid tumor. Materials Science \& Engineering $C, 113$.

Wang, X., He, L., Wei, B., Yan. G., Wang, J., \& Tang, R. (2018). Bromelain-immobilized and lactobionic acid-modified chitosan nanoparticles for enhanced drug penetration in tumor tissues. International Journal of Biological Macromolecules, 115, 129-142. 